\title{
On evolutionary ray-projection dynamics
}

\author{
Reinoud Joosten • Berend Roorda
}

Received: 25 September 2009 / Accepted: 7 December 2010 / Published online: 4 January 2011 (C) The Author(s) 2011. This article is published with open access at Springerlink.com

\begin{abstract}
We introduce the ray-projection dynamics in evolutionary game theory by employing a ray projection of the relative fitness (vector) function, i.e., a projection unto the unit simplex along a ray through the origin. Ray-projection dynamics are weakly compatible in the terminology of Friedman (Econometrica 59:637-666, 1991), each of their interior fixed points is an equilibrium and each interior equilibrium is one of its fixed points. Furthermore, every interior evolutionarily stable strategy is an asymptotically stable fixed point, and every strict equilibrium is an evolutionarily stable state and an evolutionarily stable equilibrium. We also employ the ray-projection on a set of functions related to the relative fitness function and show that several well-known evolutionary dynamics can be obtained in this manner.
\end{abstract}

Keywords Evolutionary games · Ray-projection dynamics ·

Dynamic and evolutionary stability

\section{Introduction}

We introduce a class of dynamics to model evolutionary changes in game theory. We draw inspiration from rather early literature on price-adjustment processes as introduced by Samuelson (1941) and subsequent results by Arrow and Hurwicz (1958), Arrow and Hurwicz (1960a,b) and Arrow et al. (1959). Our second source of inspiration

We thank two referees, Ulrich Witt and audiences in Tilburg, Amsterdam and Stony Brook for comments and suggestions for improvement.

R. Joosten $(\varangle) \cdot$ B. Roorda

School of Management \& Governance, University of Twente, POB 217, 7500 AE Enschede,

The Netherlands

e-mail: r.a.m.g.joosten@utwente.nl 
is recent work featuring projection dynamics, e.g., Lahkar and Sandholm (2008), Hofbauer and Sandholm (2009).

In the latter papers it is shown that if a so-called stable game possesses an interior evolutionarily stable state [ESS, Maynard Smith and Price (1973)], the so-called projection dynamics converge to it from any starting point. In fact, the proofs imply that for these dynamics every interior evolutionarily stable state is an evolutionarily stable equilibrium [ESE, Joosten (1996)], i.e., trajectories converge to the equilibrium and along any such trajectory the Euclidean distance to it decreases strictly in time.

In the literature on price-adjustment processes, a similar result ${ }^{1}$ was established about half a century ago, see e.g., Uzawa (1961), Negishi (1962). If the Weak Axiom of Revealed Preferences [WARP, Samuelson (1938)] holds, the price-adjustment process, or tâtonnement, of Samuelson (1941) given by

$$
\dot{x}=\frac{d x}{d t}=f(x) \text { for all } x \in \mathbb{P}=\mathbb{R}_{+}^{n+1} \backslash\left\{0^{n+1}\right\},
$$

converges to an economic equilibrium. Here, $x$ denotes a vector of prices for $n+1$ commodities in the price space $\mathbb{P}=\mathbb{R}_{+}^{n+1} \backslash\left\{0^{n+1}\right\}, 0^{n+1}$ denotes the $n+1$-vector of zeros, and the (vector) function $f: \mathbb{P} \rightarrow \mathbb{R}^{n+1}$ is an excess demand function. An excess demand function gives for each commodity the difference between its demand and supply given a price for all commodities. An equilibrium is a price vector for which there exists no positive excess demand for any commodity, i.e., $y$ is an equilibrium iff $f(y) \leq 0^{n+1}$. A fixed point of the dynamics is a price vector for which the time derivative under the dynamics evaluated at that state equals zero in all components, i.e., $y$ is a fixed point iff $\dot{y}=0^{n+1}$.

Our basic idea is to project a(ny) trajectory of Samuelson's tâtonnement process in $\mathbb{P}$ on the $n$-dimensional unit simplex such that every point of the original is projected on the unit simplex along the ray through this point and the origin. By the convergence result of the unrestricted dynamics under WARP mentioned, it follows that the projected dynamics also converge to an equilibrium. We show that the ray-projection dynamics of Samuelson's tâtonnement process on the unit simplex after a nonlinear time-transformation, for every $x \in$ int $\Delta^{n}$, given by

$$
\dot{x}=f(x)-x \sum_{i=1}^{n+1} f_{i}(x)
$$

where $\Delta^{n}=\left\{z \in \mathbb{R}^{n+1} \mid z_{j} \geq 0\right.$ for all $j \in\{1,2, \ldots, n+1\}$ and $\left.\sum_{j=1}^{n+1} z_{j}=1\right\}$.

\footnotetext{
${ }^{1}$ For analogies between models, concepts, results and dynamics in economics and biology, see Joosten $(1996,2006)$. For instance, a Walrasian equilibrium in economics is equivalent to a Nash equilibrium in an evolutionary game. Moreover, an implication of WARP in economics is equivalent to an implication of ESS in evolutionary game theory.
} 
The orthogonal-projection dynamics of Lahkar and Sandholm (2008), as we will call them, are for $x \in$ int $\Delta^{n}$ given by

$$
\dot{x}=f(x)-\frac{1}{n+1} 1^{n+1} \sum_{i=1}^{n+1} f_{i}(x) .
$$

Here, $f: \Delta^{n} \rightarrow \mathbb{R}^{n+1}$ is a relative fitness function [cf., Joosten (1996)], and $1^{n+1}=$ $(1, \ldots, 1)^{\top} \in \mathbb{R}^{n+1}$.

We define our evolutionary ray-projection dynamics by (2) where $f$ is a relative fitness function. We prove that under these dynamics every interior ESS is an asymptotically stable fixed point. We also show that the concept of a strict saturated equilibrium unifies two notions of evolutionary stability, namely static evolutionary stability as embodied by the ESS and dynamic evolutionary stability as embodied by ESE.

We extend the dynamics to the boundary of the state space and define rayprojection dynamics for modifications of the relative fitness function. As it turns out, the dynamics of Brown and von Neumann (1950), the logit dynamics of Fudenberg and Levine (1998), but also the replicator dynamics of Taylor and Jonker (1978), can be represented as ray-projection dynamics by choosing appropriate variants of the relative fitness function.

Next, we present our ideas leading to the ray-projection dynamics. Section 3 extends the scope of ray-projection dynamics, Sect. 4 concludes, all proofs are to be found in the Appendix.

\section{Comparing the old and the new}

We first give a very concise introduction on pure exchange economies and priceadjustment dynamics, then we show that the price-adjustment dynamics of Samuelson (1941) can be projected on the unit simplex and we provide explicit formulas for these projected dynamics. Next, we give a very brief introduction on dynamics and equilibria in evolutionary game theory to continue with projection dynamics in an evolutionary framework; we discuss the dynamics of Lahkar and Sandholm (2008) and propose our own variant of projection dynamics as evolutionary dynamics. The final subsection is devoted to stability of interior equilibria. The reader interested beyond what is presented, is referred to e.g., Joosten $(1996,2006)$.

\subsection{On price-adjustment dynamics}

The condition implied by WARP, cf., e.g., Uzawa (1961), is the following

$$
(y-x) \cdot f(x)>0,
$$

for all $x, y \in \mathbb{P}=\mathbb{R}_{+}^{n+1} \backslash\left\{0^{n+1}\right\}$ such that $\left.y \in E=\{z \in \mathbb{P}\} \mid f(z) \leq 0^{n+1}\right\}, x \notin E$. Here, $f: \mathbb{P} \rightarrow \mathbb{R}^{n+1}$ satisfies continuity, homogeneity (of degree zero in prices), i.e., $f(\lambda x)=f(x)$ for all $\lambda>0$, and complementarity, i.e., $x \cdot f(x)=0$ for all 
$x \in \mathbb{P}$. Often, since the function $f$ satisfies homogeneity of degree zero, analysis is restricted to the $n$-dimensional unit simplex $\Delta^{n}=\left\{x \in \mathbb{P} \mid \sum_{j \in I^{n+1}} x_{j}=1\right\}$, where $I^{n+1}=\{1, \ldots, n+1\}$.

In economics, $x \in \Delta^{n}$ represents a vector of relative prices adding up to unity; the function $f$ represents a so called generalized excess demand function. A price vector $y \in \Delta^{n}$ satisfying $f(y) \leq 0^{n+1}$ is called an equilibrium or a Walrasian equilibrium. At an equilibrium no commodity has positive excess demand. Existence of an equilibrium (ray) is readily shown by using homogeneity in order to restrict analysis to $\Delta^{n}$, constructing an adequate continuous function from $\Delta^{n}$ unto itself, and then using Brouwer's fixed point theorem. A generalized excess demand function on the unit simplex satisfies continuity and complementarity.

A well-known result by Arrow and Hurwicz (1958, 1960a,b), Arrow et al. (1959) is that the tâtonnement process of Samuelson (1941):

$$
\dot{x}=\frac{d x}{d t}=f(x)
$$

converges to an equilibrium if $(y-x) \cdot f(x)>0$ for all $y \in E$, and $x \notin E$ and if desirability holds. Here, $E=\left\{x \in \mathbb{R}^{n+1} \mid f(x) \leq 0^{n+1}\right\}$ denotes the set of (economic) equilibria, and if the condition mentioned holds, it can be shown that $E$ is convex [cf., Arrow and Hurwicz (1960b)]. Moreover the Euclidean distance to the set of equilibrium price vectors decreases monotonically in time along any trajectory started in a non-equilibrium price vector.

\subsection{Ray-projection of Samuelson's tâtonnement process}

Now, we derive the dynamics (2) as the projection of Samuelson's tâtonnement process on the unit simplex. Note that the trajectory $\left\{y_{t}\right\}_{t \geq 0}$ with $y_{0} \in \mathbb{P}$ under (1) may be approximated at $y \in\left\{y_{t}\right\}_{t \geq 0}$ by $y+\Delta t f(y)$. Let the projection of $y$, and $y+\Delta t f(y)$ unto the unit simplex $\Delta^{n}$ be given by

$$
x=\frac{y}{\sum_{i=1}^{n+1} y_{i}} \text { and } \tilde{x}=\frac{y+\Delta t f(y)}{\sum_{i=1}^{n+1} y_{i}+\Delta t \sum_{i=1}^{n+1} f_{i}(y)} .
$$

So, $\Delta t$ is the length of the time interval elapsed, $\sum_{i=1}^{n+1} y_{i}+\Delta t \sum_{i=1}^{n+1} f_{i}(y)$ is a number, whereas $y$ and $f(y)$ are vectors. Then, this implies a move from $x \in \Delta^{n}$ to $\widetilde{x} \in \Delta^{n}$ and therefore

$$
\begin{gathered}
\Delta x=\tilde{x}-x \stackrel{y=\lambda x}{=} \frac{\lambda x+\Delta t f(\lambda x)}{\sum_{i=1}^{n+1} \lambda x_{i}+\Delta t \sum_{i=1}^{n+1} f_{i}(\lambda x)}-\frac{\lambda x}{\sum_{i=1}^{n+1} \lambda x_{i}} \\
\sum_{i=1}^{n+1} \lambda x_{i}=\lambda
\end{gathered}
$$




$$
\begin{aligned}
& =\frac{\lambda x+\Delta t f(\lambda x)-x\left(\lambda+\Delta t \sum_{i=1}^{n+1} f_{i}(\lambda x)\right)}{\lambda+\Delta t \sum_{i=1}^{n+1} f_{i}(\lambda x)} \\
& \stackrel{f(\lambda x)=f(x)}{=} \Delta t \frac{f(x)-x \sum_{i=1}^{n+1} f_{i}(x)}{\lambda+\Delta t \sum_{i=1}^{n+1} f_{i}(x)} .
\end{aligned}
$$

So, this means that

$$
\dot{x}=\lim _{\Delta t \downarrow 0} \frac{\Delta x}{\Delta t}=\lim _{\Delta t \downarrow 0} \frac{\Delta t}{\Delta t} \frac{f(x)-x \sum_{i=1}^{n+1} f_{i}(x)}{\lambda+\Delta t \sum_{i=1}^{n+1} f_{i}(x)}=\frac{1}{\lambda}\left[f(x)-x \sum_{i=1}^{n+1} f_{i}(x)\right] .
$$

The direction of the dynamics are not influenced by the term $\frac{1}{\lambda}$, hence a transformation of time allows its omission. This motivated the following.

Definition 1 Let $f: \mathbb{P} \rightarrow \mathbb{R}^{n+1}$ satisfy continuity, complementarity, and (positive) homogeneity of degree zero. Let for all $y \in \mathbb{P}, \dot{y}=\frac{d y}{d t}=f(y)$. Then, the rayprojection dynamics on the unit simplex are for every $x=\frac{1}{\sum_{i=1}^{n+1} y_{i}} y \in i n t \Delta^{n}$ given by $\dot{x}=f(x)-x \sum_{i=1}^{n+1} f_{i}(x)$.

Here, we are not concerned with the behavior of these dynamics on the boundary of the unit simplex (boundary behavior is treated in Sect. 3).

\subsection{On dynamics and equilibria in evolutionary game theory}

In evolutionary game theory, for a population having $n+1$ distinguishable subgroups, $x \in \Delta^{n}$ is a vector of population shares for each subgroup. Let $F: \Delta^{n} \rightarrow \mathbb{R}^{n+1}$ be a fitness function, i.e., a function attributing to each subgroup in the population its fitness. The fitness of a subgroup may be interpreted as its potential to reproduce depending on the composition of the population.

The relative fitness function $f: \Delta^{n} \rightarrow \mathbb{R}^{n+1}$ is given by

$$
f_{i}(x)=F_{i}(x)-x \cdot F(x) \text { for all } \quad x \in \Delta^{n} \text { and all } i \in I^{n+1} .
$$

So, a relative fitness function [cf., Joosten (1996)] attributes to each subgroup the difference between its fitness and the population share weighted average fitness of the population. Continuity of $F$ immediately implies continuity of $f$. Observe furthermore that for all $x \in \Delta^{n}$, it holds that $x \cdot f(x)=0$.

The evolution of the composition of the population is usually represented by a system of $n+1$ autonomous differential equations:

$$
\dot{x}=\frac{d x}{d t}=h(x) \text {. }
$$

Here, the function $h: \Delta^{n} \rightarrow \mathbb{R}^{n+1}$ is connected to the relative fitness function $f$ in one of the ways proposed, cf., e.g., Nachbar (1990), Friedman (1991), Swinkels 
(1993), Joosten (1996), Ritzberger and Weibull (1995). (Lipschitz) continuity of $h$ implies existence (and uniqueness) of a solution to the differential equation for every starting point; differentiability of $h$ implies both existence and uniqueness [cf., e.g., Perko (1991)]. We do not impose conditions on the function $h$ at this point since many interesting evolutionary dynamics are neither differentiable, nor continuous.

For sign-compatible dynamics, we have

$$
\operatorname{sign} h_{i}(x)=\operatorname{sign} f_{i}(x) \text { whenever } x_{i}>0 \text {. }
$$

i.e., the change in population share of each subgroup with positive population share corresponds in sign with its relative fitness. A slightly more general alternative is provided by Friedman (1991), evolutionary dynamics are weakly compatible if $f(x) \cdot h(x) \geq 0$ for all $x \in \Delta^{n}$.

The state $y \in \Delta^{n}$ is a saturated equilibrium if $f(y) \leq \mathbf{0}^{n+1}$, a fixed point if $h(y)=\mathbf{0}^{n+1}$; a fixed point $y$ is (asymptotically) stable if, for any neighborhood $U \subset \Delta^{n}$ of $y$, there exists an open neighborhood $V \subset U$ of $y$ such that any trajectory starting in $V$ remains in $U$ (and converges to $y$ ). A limit point is a point $y \in \Delta^{n}$ satisfying $\lim _{t \rightarrow \infty} x_{t}=y$ for at least one solution $\left\{x_{t}\right\}_{t \geq 0}$ to $x_{0} \in \Delta^{n}$ and the differential equation above. At a saturated equilibrium all subgroups with below average fitness have population share equal to zero. The term is due to Hofbauer and Sigmund (1988), in the sequel we often omit the term 'saturated'.

The fixed point $y \in \Delta^{n}$ is a generalized evolutionarily stable state [GESS, Joosten (1996)] if and only if there exists an open neighborhood $U \subset \Delta^{n}$ of $y$ satisfying

$$
(y-x) \cdot f(x)>0 \text { for all } x \in U \backslash\{y\} .
$$

The GESS generalizes the concept of an ESS of Maynard Smith and Price (1973) in order to deal with arbitrary (relative) fitness functions. For the more standard fitness functions, the two notions coincide.

Joosten (1996) defined another concept as follows. The fixed point $y \in \Delta^{n}$ is an evolutionarily stable equilibrium (ESE) if and only if there exists an open neighborhood $U \subset \Delta^{n}$ of $y$ satisfying

$$
(y-x) \cdot h(x)>0 \text { for all } x \in U \backslash\{y\}
$$

The concept was inspired by the Euclidean distance approach of early contributions in economics as mentioned, since (4) implies that the (squared) Euclidean distance is a (strict) Lyapunov function for $U$.

\subsection{Projection dynamics in evolutionary games}

Lahkar and Sandholm (2008) introduce the following dynamics into evolutionary game theory. 


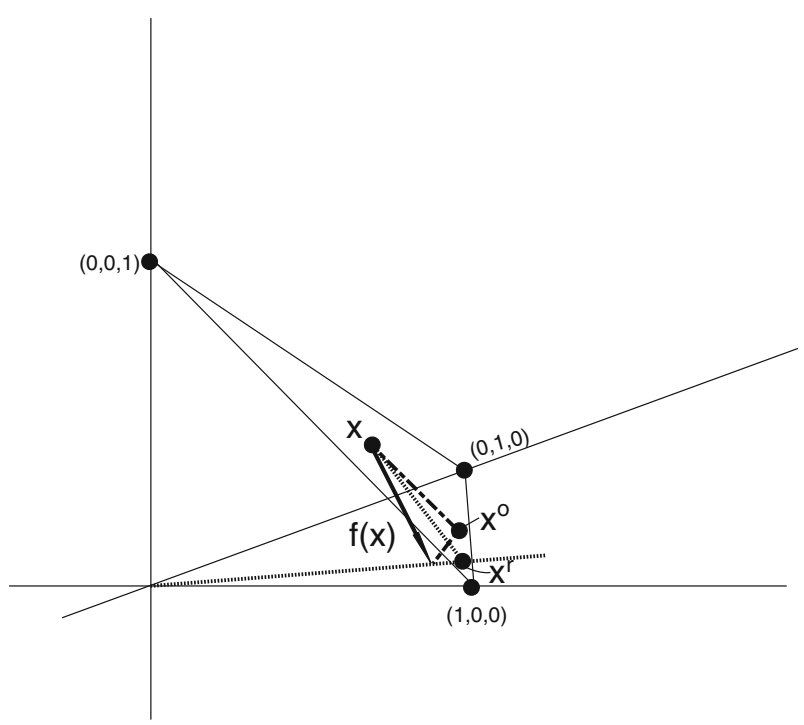

Fig. 1 The point $x^{o}$ is the orthogonal projection of $x+f(x)$ on the $S^{2} ; x^{r}$ is the ray-projection of $x+f(x)$ on $S^{n}$

Definition 2 Let $f: \Delta^{n} \rightarrow \mathbb{R}^{n+1}$ be a relative fitness function, then, the orthogonal-projection dynamics are for every $x \in$ int $\Delta^{n}$ given by: $\dot{x}=f(x)-$ $\left(\frac{1}{n+1} 1^{n+1}\right) \sum_{i=1}^{n+1} f_{i}(x)$.

Recall that the part between brackets represents a number and $1^{n+1}$ is an $(n+1)$-vector of ones. For the time being, we are only interested in the behavior of these dynamics on the interior of the unit simplex. The authors actually define their dynamics on the fitness function, but for the interior of the unit simplex their definition and the one given above concur.

Our evolutionary ray-projection dynamics can be formalized as follows. Let $f$ : $\Delta^{n} \rightarrow \mathbb{R}^{n+1}$ be a relative fitness function, then the ray-projection dynamics are for every $x \in$ int $\Delta^{n}$ given by Definition 1 , i.e.,

$$
\dot{x}=f(x)-x \sum_{i=1}^{n+1} f_{i}(x) .
$$

These dynamics are weakly-compatible in the sense of Friedman (1991).

Informally stated, both processes move from $x \in \Delta^{n}$ into the direction $f(x)$, hence outside the unit simplex in general. Lahkar and Sandholm's dynamics return to the unit simplex by continuously changing all components with identical amounts, whereas our dynamics are brought back to the unit simplex by continuously changing all components proportional to $x$, see Fig. 1 for an illustration.

For the framework presented, we have the following result. 
Proposition 1 Every interior equilibrium is a fixed point of both types of projection dynamics and every interior fixed point of both types of projection dynamics is an equilibrium.

\subsection{On stability of interior equilibria}

Hofbauer and Sandholm (2009) introduce the class of stable games in which the following property holds:

$$
(y-x) \cdot(f(y)-f(x)) \leq 0 \text { for all } x, y \in \Delta^{n} .
$$

This property is called monotonicity $(M O N)$ elsewhere and is connected to a multitude of results guaranteeing uniqueness and dynamic stability of equilibria and fixed points [see Joosten (2006)]. MON is a weaker version of strict monotonicity (SMON) and the latter can be written as

$$
(y-x) \cdot(f(y)-f(x))<0 \text { for all } x, y \in \Delta^{n}, x \neq y \text {. }
$$

A game in which $S M O N$ holds for all states $x, y \in \Delta^{n}, x \neq y$, is called a strictly stable game. It can be shown that $S M O N$ implies that there is a unique saturated equilibrium, and that $M O N$ implies that the set of equilibria is compact and convex.

For an interior equilibrium $y \in \Delta^{n},(S) M O N$ implies

$$
(y-x) \cdot f(x) \geq(>) 0 \text { for all } x \in \Delta^{n} \backslash\{y\} .
$$

So, an interior equilibrium of a strictly stable game is a GESS [cf., Joosten (1996)]. For a stable game, every interior equilibrium is a neutrally stable state [cf., Joosten (2006), Maynard Smith (1982)].

For the orthogonal-projection dynamics it can be seen that every interior evolutionarily stable equilibrium is a generalized evolutionarily stable state and every interior generalized evolutionarily stable state is an evolutionarily stable equilibrium, as for $y \in$ int $\Delta^{n}$ we have

$$
\begin{aligned}
& (y-x) \cdot h(x)>0 \\
& \Longleftrightarrow(y-x) \cdot f(x)-(y-x)\left(\frac{1}{n+1} 1^{n+1}\right) \sum_{i=1}^{n+1} f_{i}(x)>0 \\
& \Longleftrightarrow(y-x) \cdot f(x)>0 .
\end{aligned}
$$

We now present a corresponding result for ray-projection dynamics.

Proposition 2 Under the ray-projection dynamics, every interior generalized evolutionarily stable state is an asymptotically stable fixed point. 


\section{Extending the scope for projection dynamics}

In this section we extend the scope for projection dynamics into two directions. First, we propose several manners in which to extend the dynamics from the interior of the unit simplex to its boundary. Second, we pursue the idea of replacing the relative fitness functions in the definitions of projection dynamics by other functions which, in order to make sense in an evolutionary framework, should be connected to the relative fitness function.

\subsection{To the boundary}

The standard way of dealing with Samuelson's dynamics on the boundary of $\mathbb{P}$ is to define them as being zero for every zero component of the state variable, see e.g., Arrow and Hurwicz (1958, 1960a,b), Arrow et al. (1959). In our notations the extension to include the boundary of $\mathbb{P}$ would be given by

$$
\dot{x}_{i}= \begin{cases}0 & \text { if } x_{i}=0 \\ f_{i}(x) & \text { otherwise }\end{cases}
$$

So, the dynamics extended to the boundary may be discontinuous. For the rayprojection dynamics this extension to the boundary does not pose great problems as we may (re)define

$$
\dot{x}_{i}^{r}= \begin{cases}0 & \text { if } x_{i}=0, \\ f_{i}(x)-x_{i} \sum_{j: x_{j}>0} f_{j}(x) & \text { otherwise. }\end{cases}
$$

Under (a), a trajectory might in finite time reach the boundary of the unit simplex, and then remain on it (even if the relative fitness of a subgroup with population share zero becomes positive again).

An alternative is to define the dynamics extended as

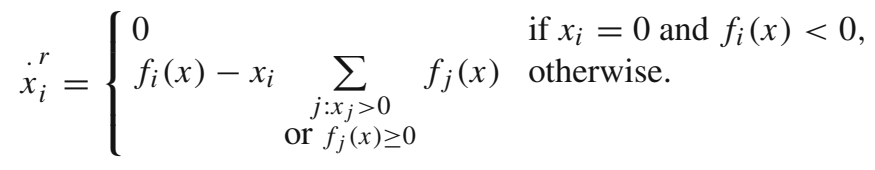

This way, the dynamics escape the boundary of $\Delta^{n}$ as soon as $f_{i}(x)>0$. So, at a limit point $y \in b d \Delta^{n}$, we can never have $y_{i}=0$ and $f_{i}(y)>0$. Note, however, that the dynamics are discontinuous at the boundary of the state space as before.

A final way of covering the boundary conditions in an appropriate way is inspired by Nikaidô and Uzawa (1960). Let $\rho$ be a non-negative real number, then we may extend the ray-projection dynamics to the boundary in the following manner 


$$
\dot{x}_{i}^{r}=\lim _{\rho \downarrow 0} \frac{1}{\rho}\left[\max \left\{0, \rho f_{i}(x)+x_{i}\right\}-x_{i} \sum_{j=1}^{n+1} \max \left\{0, \rho f_{j}(x)+x_{j}\right\}\right] .
$$

These dynamics have non-negative sign at the boundary of the state space. Moreover, the discontinuity problem is solved and on a very large subset of the state space, the dynamics are equivalent to the original.

The following result has interesting implications. Let, $Z P=\left\{x \in \Delta^{n} \mid f(x)=\right.$ $\left.0^{n+1}\right\}, F P=\left\{x \in \Delta^{n} \mid \dot{x}=0^{n+1}\right\}$ and note that $Z P \subseteq(F P \cap E)$.

Proposition 3 Let $\left\{x_{t}\right\}_{t \geq 0}$ be a trajectory under the ray-projection dynamics and let $y=\lim _{t \rightarrow \infty} x_{t}$. If $t^{*}$ exists such that $\left\{x_{t}\right\}_{t \geq t^{*}} \subset$ int $\Delta^{n}$, then $y \in Z P$; otherwise, $y \in b d \Delta^{n}$ and under (a) $y \in F P$, under (b or c) $y \in E$.

Boundary conditions are of high relevance for boundary equilibria, fixed points and limit points. A refinement of the saturated equilibrium concept is the strict saturated equilibrium [cf., Joosten (1996)] which is a saturated equilibrium satisfying $f_{j}(y)=0$ for precisely one $j \in I^{n+1}$. For this type of equilibrium we have the following result and in its proof the exact details about the boundary conditions become obsolete.

Proposition 4 Every strict saturated equilibrium is an evolutionarily stable equilibrium of the ray-projection dynamics.

\subsection{To functions connected to the relative fitness function}

Let $g: \mathbb{R}^{n+1} \rightarrow \mathbb{R}^{n+1}$, and take the ray-projection dynamics induced by $g$ as follows:

$$
\dot{x}_{g}=g(x)-x \sum_{i=1}^{n+1} g_{i}(x)
$$

Then, the following straightforward observations are useful.

- If $g$ is weakly compatible with $f$, i.e., $g(x) \cdot f(x) \geq 0$ for all $x \in$ int $\Delta^{n}$, then the associated dynamics are weakly compatible.

- If $g$ is non-negative, i.e., $g: \mathbb{R}^{n+1} \rightarrow \mathbb{R}_{+}^{n+1}$, then the dynamics remain on the unit simplex.

The first observation gives a criterion to determine the status of the ensuing dynamics. Recall that evolutionary dynamics should be connected with the relative fitness function. Weak compatibility of Friedman (1991) is one of the ways to accomplish this. Hence, if $g$ is sign-compatible with $f$, then the resulting ray-projection dynamics are weakly compatible. The second one deals with a criterion to guarantee that ray-projection dynamics do not cross the boundary of the unit simplex.

In order to be relevant in an evolutionary framework it is of utmost importance to link the function $g$ to the relative fitness function. It is not the purpose of this section to give a classification of functions suitable for evolutionary modeling purposes. Instead we show that several well-known dynamics can be represented as ray-projection dynamics, allowing a unified analysis of important evolutionary dynamics. 
Example 1 Let $g:$ int $\Delta^{n} \rightarrow \mathbb{R}^{n+1}$ be given (componentwise) by

a. $g_{i}(x)=x_{i} F_{i}(x)$,

b. $g_{i}(x)=z\left(\max \left\{0, f_{i}(x)\right\}\right)$ where $z: \mathbb{R}_{+} \rightarrow \mathbb{R}_{+}$is continuous and satisfies $z(0)=0$ and $z(x)>0$ for all $x>0$,

c. $g_{i}(x)=\max \left\{0, \rho f_{i}(x)+x_{i}\right\}-x_{i}$ where $\rho>0$,

d. $g_{i}(x)=e^{\beta f_{i}(x)}$ where $\beta>0$,

e. $g_{i}(x)=x_{i} e^{\beta f_{i}(x)}$ where $\beta>0$.

(a) Here, ray-projection yields the replicator dynamics of Taylor and Jonker (1978). For these weakly compatible dynamics, each interior equilibrium is a fixed point and vice versa, also all boundary equilibria are fixed points.

(b) These ray-projections were called 'Brownian motions' in Hofbauer (2000) after G.W. Brown (not R. Brown, the (re)discoverer of Brownian motion). If $z(x)=x^{\alpha}$ for $\alpha>0, x \geq 0$, then $\alpha=1$ yields the dynamics of Brown and von Neumann (1950), or BN-dynamics. For $\alpha \rightarrow \infty$, the dynamics are a continuous version of the best-response dynamics of Matsui (1992), or BR-dynamics, which have a predecessor in the dynamics of Rosenmüller (1971), a continuous-time version of fictitious play (Brown 1951). For these weakly compatible 'Brownian motions', each interior equilibrium is a fixed point and vice versa, and all boundary equilibria are fixed points as well.

(c) This variant yields another 'Brownian motion'. The function is used by Nikaidô and Uzawa (1960) in the framework of price-adjustment. The ray-projection dynamics are weakly compatible and each interior equilibrium is a fixed point and vice versa. All boundary equilibria are fixed points, too.

(d) Here, the ray-projection dynamics are (up to a nonlinear transformation of time) equivalent to the logit dynamics (Fudenberg and Levine 1998). For $\beta \rightarrow \infty$ these dynamics become similar to the BR-dynamics, but remain continuous. A glaring shortcoming of the logit dynamics is that an interior equilibrium need not be a fixed point of the dynamics. Clearly, the ray-projection dynamics do not cross the boundary of $\Delta^{n}$.

(e) Here, the ray-projection dynamics are equivalent to those of Björnerstedt and Weibull (1996). Cabrales and Sobel (1992) have presented a discrete-time version. Note that the ray-projection does not cross the boundary $\Delta^{n}$.

For the replicator dynamics, Taylor and Jonker (1978) provided conditions guaranteeing that each ESS is an asymptotically stable fixed point; Zeeman (1981) extended this result; the most general stability result on these dynamics and the ESS is probably Hofbauer et al. (1979).

$\mathrm{BN}$-dynamics converge to a Nash equilibrium for zero-sum games. Moreover, BN-dynamics are globally stable under strict monotonicity (SMON) of the generalized excess demand function [cf., Nikaidô (1959)]. Hofbauer (2000) treats families of dynamics including (smoothed) BN-dynamics, BR-dynamics and replicator dynamics. His convergence results on the ESS complement Nikaidô's. Stability of the ESS for the logit dynamics is shown in Hopkins (1999) and Hofbauer (2000). 
Nikaidô and Uzawa (1960) show that any interior equilibrium is asymptotically stable for their dynamics under WARP. For $\rho \rightarrow+\infty$ the ray-projection of their process 'approximates' the BN-dynamics 'almost everywhere'; for $\rho \downarrow 0$ the ray-projection dynamics are equivalent to the ray-projection of Samuelson's process 'almost everywhere'. Clearly, for any interior equilibrium, there exists a neighborhood such that the latter two concur. So, any interior ESS is an asymptotically stable fixed point of the ray-projection of the dynamics of Nikaidô and Uzawa.

\section{Conclusions}

We introduced new evolutionary dynamics in game theory, the ray-projection dynamics. We have shown that every interior (generalized) evolutionarily stable strategy is an asymptotically stable fixed point of the ray-projection dynamics. We showed that each strict saturated (Hofbauer and Sigmund 1988) equilibrium is both a generalized evolutionarily stable state [Joosten (1996) after Maynard Smith and Price (1973)] and an evolutionarily stable equilibrium (Joosten 1996) for ray-projection dynamics.

We applied projections to dynamics driven by functions connected to the relative fitness function. It turns out that well-known dynamics in evolutionary game theory can be represented as projection dynamics for appropriately chosen functions. Even if well-known dynamics can not be recovered in full, attractive elements may be used for new projection dynamics. For instance, the generalized replicator dynamics of Sethi (1998) introduced in a learning framework in which strategies are not equally easily adopted, can not be recovered by ray projection. Yet, the 'inflows' incorporating the possible differences in which strategies can be adopted, can be taken to motivate new evolutionary dynamics.

The strategy of proof for our first major result contains some promise for future research. We transformed a dynamic process on the unit simplex into a dynamic process in the positive orthant, then projected the latter unto the unit simplex. We took a known result on price-adjustment dynamics in the positive orthant to show stability of the unrestricted dynamics, i.e., convergence to an equilibrium ray, implying the same properties for the connected ray-projection dynamics on the unit simplex. There is an abundance of stability results on both restricted and unrestricted tâtonnements [cf., e.g., Uzawa (1961), Negishi (1962)] which may be used to derive stability results for evolutionary dynamics using a similar strategy of proof. In this context, an important topic for further research is to find a classification for the functions admissible for projection.

Microfoundations were not a theme of this paper, but connections between the ones given by e.g., Lahkar and Sandholm (2008) seem immediate. Tsakas and Voorneveld (2009) show that target-projection dynamics (Sandholm 2005) can be associated to rational choice behavior if control costs [as in e.g., Van Damme (1991)] can be assumed [see also Mattson and Weibull (2002), Voorneveld (2006)]. Further research must reveal which dynamics can be motivated with such microeconomic foundations.

Open Access This article is distributed under the terms of the Creative Commons Attribution Noncommercial License which permits any noncommercial use, distribution, and reproduction in any medium, provided the original author(s) and source are credited. 


\section{Appendix}

Proof of Proposition 3 The part 'interior equilibrium implies fixed point' is evident. Conversely, let $y \in$ int $\Delta^{n}$ be a fixed point of the ray-projection dynamics. Then, $f_{i}(y)-y_{i}\left(\sum_{j=1}^{n+1} f_{j}(y)\right)=0$ for all $i \in I^{n+1}$. This in turn implies $y_{i} f_{i}(y)=$ $y_{i}^{2}\left(\sum_{j=1}^{n+1} f_{j}(y)\right)$ for all $i \in I^{n+1}$. Then, summing over all $i \in I^{n+1}$ and complementarity of $f$ lead to $0=y \cdot f(y)=\sum_{i=1}^{n+1} y_{i}^{2}\left(\sum_{j=1}^{n+1} f_{j}(y)\right)$. This can only hold if $\sum_{j=1}^{n+1} f_{j}(y)=0$, hence $f(y)=0^{n+1}$. For orthogonal-projection dynamics, the reasoning is similar.

Proof of Proposition 4 Let $f: \Delta^{n} \rightarrow \mathbb{R}^{n+1}$ be a continuous relative fitness function. Define $\tilde{f}: \mathbb{P} \rightarrow \mathbb{R}^{n+1}$ by $\tilde{f}(\lambda x)=f(x)$ for all $\lambda>0$. Then, $\tilde{f}$ is continuous, homogeneous of degree zero, and satisfies complementarity. Define for all $x \in \mathbb{P}$ :

$$
\dot{x}=\widetilde{f}(x) .
$$

Clearly, this implies that $\frac{d\|x\|^{2}}{d t}=2 \sum_{j=1}^{n+1} x_{j} \dot{x}_{j}=2 \sum_{j=1}^{n+1} x_{j} \widetilde{f}_{j}(x)=0$. Let $\left\{x_{t}\right\}_{t \geq 0}$ denote a solution to $x_{0} \in \mathbb{P}$ and Eq. (5). Then, $\left\{x_{t}\right\}_{t \geq 0}$ remains on the sphere with the origin as center and with radius $r=\left\|x_{0}\right\|$.

Let $y \in \Delta^{n}$ be an interior generalized evolutionarily stable state, i.e., an open neighborhood $U \subseteq$ int $\Delta^{n}$ containing $y$ exists such that $(y-x) \cdot f(x)>0$ for all $x \in U \backslash\{y\}$. Let $E=\{x \in \mathbb{P} \mid x=\lambda y, \lambda>0\}$. Define for $z \in \mathbb{P}, \lambda_{z}=$ $\sum_{k=1}^{n+1} z_{k}$. Then, let $x^{*} \in \mathbb{P}$ satisfy $\frac{1}{\lambda_{x^{*}}} x^{*} \in U \backslash\{y\}$ and let $y^{*} \in E$ such that $\left\|x^{*}\right\|=\left\|y^{*}\right\|$. Then, obviously $d\left(x^{*}, y^{*}\right)^{2}>0, d\left(y^{*}, y^{*}\right)^{2}=0$ and under (5): $\frac{1}{2} d\left(x, y^{*}\right)^{2}=-\sum_{j=1}^{n+1}\left(y_{j}^{*}-x_{j}^{*}\right) \widetilde{f}_{j}\left(x^{*}\right)=-\sum_{j=1}^{n+1}\left(\lambda_{y^{*}} y_{j}-\lambda_{x^{*}} x_{j}\right) \widetilde{f}_{j}\left(\lambda_{x^{*}} x\right)=$ $-\sum_{j=1}^{n+1}\left(\lambda_{y^{*}} y_{j}-\lambda_{y^{*}} x_{j}+\left(\lambda_{y^{*}}-\lambda_{x^{*}}\right) x_{j}\right) f_{j}(x)=-\lambda_{y^{*}}(y-x) \cdot f(x)<0$. This means that the squared (Euclidean) distance is a strict Lyapunov function for $U^{\prime}=$ $\left\{x \in \mathbb{P} \mid \frac{1}{\lambda_{x}} x \in U\right\}$. Hence, an open neighborhood $U^{\prime \prime}$ of $y^{*}$ exists such that every trajectory $\left\{x_{t}\right\}_{t \geq 0}$ with $x_{0} \in U^{\prime \prime} \backslash\left\{y^{*}\right\}$ such that $\left\|x_{0}\right\|=\left\|y^{*}\right\|$, converges to $y^{*}$, i.e., $\lim _{t \rightarrow \infty} x_{t}=y^{*}$.

The ray-projection $\left\{x_{t}^{\prime}\right\}_{t \geq 0}$ of such a trajectory $\left\{x_{t}\right\}_{t \geq 0}$ with $x_{0} \in U^{\prime \prime} \backslash\left\{y^{*}\right\}$ such that $\left\|x_{0}\right\|=\left\|y^{*}\right\|$, and $\lim _{t \rightarrow \infty} x_{t}=y^{*}$ is given by $x^{\prime}=\frac{x_{0}}{\sum_{j=1}^{n+1}\left(x_{0}\right)_{j}}$ and

$$
\dot{x^{\prime}}=\frac{1}{\lambda_{x}}\left[f(x)-x \sum_{i=1}^{n+1} f_{i}(x)\right] \text { for every } x \in\left\{x_{t}\right\}_{t \geq 0} .
$$

Clearly, $\lim _{t \rightarrow \infty} x_{t}^{\prime}=y$. As the factor $\frac{1}{\lambda_{x}}$ only influences the speed of the dynamics but not the direction, it follows that any trajectory $\left\{x_{t}\right\}_{t \geq 0}$ with $x_{0} \in U^{\prime \prime \prime}$ converges to $y$ under the ray-projection dynamics given by 


$$
\dot{x}=f(x)-x \sum_{i=1}^{n+1} f_{i}(x) .
$$

So, $y$ is an asymptotically stable fixed point for (6).

Proof of Proposition 5 Let $h: \Delta^{n} \rightarrow \mathbb{R}^{n+1}$ be given by $h(x)=f(x)-x \sum_{j=1}^{n+1} f_{j}(x)$ for all $x \in \Delta^{n}$. Clearly, $h$ is continuous because $f$ is continuous on $\Delta^{n}$.

(1) Let $\left\{x_{t}\right\}_{t \geq 0}$ satisfy that some $t^{*}$ exists such that $\left\{x_{t}\right\}_{t \geq t^{*}} \subset$ int $\Delta^{n}$ and $\lim _{t \rightarrow \infty} x_{t}=y$. If $y \in$ int $\Delta^{n}$, then by continuity of $h$ it follows that $h(y)=0^{n+1}$. So, Proposition 3 applies, i.e., $y \in Z P \subseteq E$. If on the other hand, $y \in b d \Delta^{n}$, let $y_{j}=0$ and assume $f_{j}(y)>0$. By continuity of $h$ we have $h_{j}(y)>0$, and an open neighborhood $U \ni y$ exists such that $h_{j}(x)>0$ for all $x \in U$. However, since $y_{j}=0$ and $x_{j}>0$ for all $x \in\left\{x_{t}\right\}_{t \geq t^{*}}$ a subsequence $\left\{x_{t_{k}}\right\}_{k \in \mathbb{N}} \subseteq\left\{x_{t}\right\}_{t \geq t^{*}}$ must exist such that $\left(x_{t_{k}}\right)_{j}=h_{j}\left(x_{t_{k}}\right)<0$ for all $k \in \mathbb{N}$. Since $\lim _{k \rightarrow \infty} x_{t_{k}}=y,\left\{x_{t_{k}}\right\}_{k \in \mathbb{N}} \cap U \neq \varnothing$. This yields a contradiction. Hence, $y_{j}=0$ implies $f_{j}(y) \leq 0$. Furthermore, for $y_{j}>0$ we have $h_{j}(y)=0=f_{j}(y)-y_{j}\left(\sum_{k=1}^{n+1} f_{k}(y)\right)$ which by continuity implies $f_{j}(y)=y_{j}\left(\sum_{k=1}^{n+1} f_{k}(x)\right)$. However, then $0=\sum_{j: y_{j}>0} y_{j} f_{j}(y)=$ $\sum_{j: y_{j}>0} y_{j}^{2}\left(\sum_{k=1}^{n+1} f_{k}(x)\right)$, hence $\sum_{k=1}^{n+1} f_{k}(x)=0$ and $f(y)=0^{n+1}$.

(2) Suppose $\left\{x_{t}\right\}_{t \geq 0} \stackrel{t \rightarrow \infty}{\rightarrow} y$ and it does not hold that $t^{*}$ exists such that $\left\{x_{t}\right\}_{t \geq t^{*}} \subset$ int $S^{n}$. Let $T=\left\{k \in I^{n+1} \mid y_{k}>0\right.$ or $\left[y_{k}=0\right.$ and $\left(x_{t}\right)_{k}>0$ for all $t>t^{\prime}$ for some $\left.\left.t^{\prime} \geq 0\right]\right\}$. It follows from the above that for $k \in T$ it must hold that $f_{k}(y)=0$. Now, let $h \in I^{n+1} \backslash T$ then $y_{h}=\left(x_{t}\right)_{h}=0$. If (a) holds, then $\dot{x}_{h}=0$ regardless the sign of $f_{h}(x)$, hence $y \in F P$. Under (b and c), $\dot{x}_{h}>0$ whenever $f_{h}(x)>0$ and $x_{h}=0$, therefore $y \in E$.

Proof of Proposition 6 Let $y$ be a strict saturated equilibrium, then $m=\max _{h \neq j}$ $f_{h}(y)<0$ and continuity implies that a neighborhood $U \ni y$ exists such that $\max _{h \neq j} f_{h}(x) \leq \frac{m}{2}$ for all $x \in U$. Complementarity implies $y=e_{j}$. Let $C_{S}(x)=\sum_{h \in S \cup\{j\}} f_{h}(x)$ for $\varnothing \neq S \subseteq I^{n+1} \backslash\{j\}$. Then, clearly $C_{S}(y) \leq m<0$ for all nonempty $S \subseteq I^{n+1} \backslash\{j\}$ and a neighborhood $U^{\prime} \ni y$ exists such that $\max _{S \subseteq I^{n+1} \backslash\{j\}} C_{S}(x) \leq \frac{m}{2}$ for all $x \in U^{\prime}$. Next, let $x \in U \cap U^{\prime}$, then $(y-x) \cdot \dot{x}=$ $\left(e_{j}-x\right) \cdot f(x)-C_{S^{\prime}}(x)\left(e_{j}-x\right) \cdot x \geq-\frac{\left(\sum_{h \neq j} x_{h} f_{h}(x)\right)}{x_{j}}-\left(x_{j}-x \cdot x\right) \frac{m}{2} \geq-\frac{1-x_{j}}{x_{j}} \frac{m}{2}-$ $\left(1-x_{j}\right) \frac{m}{2}\left(x_{j}-\max _{h \neq j} x_{h}\right)=-\left(1-x_{j}\right) \frac{m}{2}\left(\frac{1}{x_{j}}+\left(x_{j}-\max _{h \neq j} x_{h}\right)\right) \geq$ $-\left(1-x_{j}\right) \frac{m}{2} \geq 0$. Here, we have a strict inequality whenever $x_{j} \neq 1$. So, $y \in E S E$.

\section{References}

Arrow KJ, Block HD, Hurwicz L (1959) On the stability of the competitive equilibrium II. Econometrica 27:82-109 
Arrow KJ, Hurwicz L (1958) On the stability of the competitive equilibrium I. Econometrica 26:522-552

Arrow KJ, Hurwicz L (1960a) Competitive stability under weak gross substitutability: the "Euclidean distance" approach. Int Econ Rev 1:38-49

Arrow KJ, Hurwicz L (1960b) Some remarks on the equilibria of economic systems. Econometrica 28:640646

Björnerstedt J, Weibull J (1996) Nash equilibrium and evolution by imitation. In: Arrow KJ et al (eds) The rational foundations of economic behavior. MacMillan, London pp 155-171

Brown GW (1951) Iterative solutions of games by fictitious play. In: Koopmans T (ed) Activity analysis of production and allocation. Wiley, NY, pp 374-376

Brown GW, von Neumann J (1950) Solutions of games by differential equations. Annals of mathematics studies, vol 24. Princeton University Press, Princeton, 73-79

Cabrales A, Sobel J (1992) On the limit points of discrete selection dynamics. J Econ Theory 57:407-419

Friedman D (1991) Evolutionary games in economics. Econometrica 59:637-666

Fudenberg D, Levine DK (1998) The theory of learning in games. MIT Press, Cambridge

Hofbauer J (2000) From Nash and Brown to Maynard Smith: equilibria, dynamics, and ESS. Selection 1:81-88

Hofbauer J, Sandholm WH (2009) Stable games and their dynamics. J Econ Theory 144:1665-1693

Hofbauer J, Schuster P, Sigmund K (1979) A note on evolutionary stable strategies and game dynamics. J Theor Biol 81:609-612

Hofbauer J, Sigmund K (1988) The theory of evolution and dynamical systems. Cambridge University Press, Cambridge

Hopkins E (1999) A note on best response dynamics. Games Econ Behav 29:138-150

Joosten R (1996) Deterministic evolutionary dynamics: a unifying approach. J Evol Econ 6:313-324

Joosten R (2006) Walras and Darwin: an odd couple? J Evolut Econ 16:561-573

Lahkar R, Sandholm WH (2008) The projection dynamic and the geometry of population games. Games Econ Behav 64:565-590

Matsui A (1992) Best-response dynamics and socially stable strategies. J Econ Theory 57:343-362

Mattson LG, Weibull JW (2002) Probabilistic choice and procedural bounded rationality. Games Econ Behav 41:61-78

Maynard Smith J (1982) Evolution and the theory of games. Cambridge University Press, Cambridge

Maynard Smith J, Price GA (1973) The logic of animal conflict. Nature 246:15-18

Nachbar JH (1990) 'Evolutionary' selection dynamics in games: convergence and limit properties. Int J Game Theory 19:59-89

Negishi T (1962) The stability of a competitive economy: a survey article. Econometrica 30:635-669

Nikaidô H (1959) Stability of equilibrium by the Brown-von Neumann differential equation. Econometrica 27:654-671

Nikaidô H, Uzawa H (1960) Stability and non-negativity in a Walrasian tâtonnement process. Int Econ Rev 1:50-59

Perko L (1991) Differential equations and dynamical systems. Springer, Berlin

Ritzberger K, Weibull J (1995) Evolutionary selection in normal form games. Econometrica 63:1371-1399

Rosenmüller J (1971) Über die Periodizitätseigenschaften spieltheoretischer Lernprozesse. Zeitschrift für Warscheinlichkeitstheorie und verwandte Gebiete 17:259-308

Samuelson P (1938) A note on the pure theory of consumer behavior. Economica 5:61-71

Samuelson P (1941) The stability of equilibrium: comparative statics and dynamics. Econometrica 9:97120

Sandholm WH (2005) Excess payoff dynamics and other well-behaved evolutionary dynamics. J Econ Theory 124:149-170

Sethi R (1998) Strategy-specific barriers to learning and non-monotonic selection dynamics. Games Econ Behav 23:284-304

Swinkels J (1993) Adjustment dynamics and rational play in games. Games Econ Behav 5:455-484

Taylor PD, Jonker LB (1978) Evolutionarily stable strategies and game dynamics. Math Biosci 40:145-156

Tsakas E, Voorneveld M (2009) The target projection dynamic. Games Econ Behav 67:708-719

Uzawa H (1961) The stability of dynamic processes. Econometrica 29:617-631

Van Damme EEC (1991) Stability and perfection of Nash equilibria. Springer, Berlin

Voorneveld M (2006) Probabilistic choice in games: properties of Rosenthal's $t$-solutions. Int J Game Theory 34:105-121

Zeeman EC (1981) Dynamics of the evolution of animal conflicts. J Theor Biol 89:249-270 